\title{
Quantum Mechanics Allows Setting Initial Conditions at a Cosmological Singularity: Gowdy Model Example
}

\author{
S.L. Cherkas ${ }^{1 *}$ and V.L. Kalashnikov ${ }^{2}$ \\ ${ }^{1}$ Institute for Nuclear Problems, Belarus State University, Minsk, Belarus \\ ${ }^{2}$ Institute of Photonics, Vienna University of Technology, Vienna, Austria \\ Email: cherkas@inp.bsu.by
}

\begin{abstract}
It is shown that the initial conditions in the quasi-Heisenberg quantization scheme can be set at the initial cosmological singularity per se. This possibility is provided by finiteness of some quantities, namely momentums of the dynamical variables, at a singularity, in spite of infinity of the dynamical variables themselves. The uncertainty principle allows avoiding a necessity to set values of the dynamical variables at singularity, as a wave packet can be expressed through the finite momentums. Influence of the initial condition set in the singularity in such a way to a number of gravitons under a vacuum state, arising during later evolution, is investigated. It is shown that, even choosing of some special state at the singularity minimizing late time expansion rate, some amount of gravitons still appear in the late time evolution.
\end{abstract}

Keywords: Cosmological singularity, initial conditions, quantum evolution

\section{Introduction}

One of the problems of the relativistic cosmology is the formulation of the initial conditions for the universe evolution. A lot have been done in this direction concerning quantum fields at the classical uniform background [1], in particular in describing the origin of primordial inhomogeneities $[2,3]$ giving the initial conditions on the last scattering surface for the cosmic microwave background radiation (see [4] and references given herein).

The modern description of the uniform background itself includes the inflation paradigm [5-7] which besides the description of the density perturbation values, successfully solves the problems of horizon and flatness. In describing the earlier stage of evolution, one encounters the problem of the initial conditions again. The well-known Penrouse theorem [8-10] states that under quite general conditions, the initial point of the evolution should be singular.

One of the conditions of the Penrouse theorem is the energy condition, which is violated during inflation [11], but geodesics remains past uncomplete in this case also [12]. The incompleteness of geodesics tells us that there is a moment in the past (i.e. singularity) beyond which one cannot move in past direction. It seems natural to set initial conditions at this last point of the backward evolution (initial point of future evolution). This seems quite impossible, at first sight, because the dynamical quantities such as amplitudes of the matter fields and scale factor logarithm, turn to infinity at the singularity.

It is considered that near the singularity, at the Planck epoch, quantum effects are crucial. Thus, the problem of the initial conditions and the singularity should be considered at the quantum level [13-17], although one could attempt to avoid singularity at a classical level $[18,19]$.

In relation with the singularity problem we need to discuss some ways of gravity quantization. According to [20-24], the loop quantum gravity removes the singularity completely, including different types of future singularities, such as Big Rip. The absence of singularities in loop quantum gravity originates from the fact that the volume operator (and consequently the universe scale factor) has a discrete spectrum bounded below. However, there remains a problem, how to connect this discrete spectrum with the time evolution of the universe, canonical gravity quantization and, about self-consistency of the loop quantum gravity itself. Work in this directions is in progress [25].

The canonical quantization of general relativity (GR) leads to the Wheeler-DeWitt (WDW) equation $[26,27]$, which is the analog of the Schrödinger equation of the ordinary quantum mechanics. However, the equation does not contain a time variable explicitly, so one has to interpret the wave function of the 
universe in some way. For instance, one could interpret the scale factor as time the variable; though, it could not be considered as a complete solution of the problem because one needs to describe the evolution of dynamical variables including the scale factor in time, explicitly. For instance, in Ref. [24] the effective Hamiltonian has been deduced by corrections with the loop quantum gravity effects, and then it was investigated classically (i.e., to describe time evolution, the authors of Ref. [24] return to classics).

It seems more fundamental to consider the problem of singularity and the initial conditions in a quantization scheme involving the evolution in time explicitly. Such a scheme was suggested for mini- and midi- superspace models [28-30]. In ordinary quantum mechanics, Schrödinger and Heisenberg pictures are equivalent. In quantum gravity, a canonically quantized Hamiltonian of the GR cannot serve for building the Heisenberg picture, that is, the conventional Heisenberg picture does not exist. Nevertheless, one can quantize the equations of motion straightforwardly, that is, quasi-Heisenberg picture exists (Fig. 1).

In the quantization scheme of Ref. [28-30] quasi-Heisenberg operators satisfy the commutation relations obtained from the system of constraints and gauge conditions with the help of the Dirac brackets at the initial moment of time. Then it is allowed quasi-Heisenberg operators to evolve according to the equations of motion. This evolution implicitly determines time-dependent gauge fixing, defined explicitly prior to quantization only at initial moment of time.

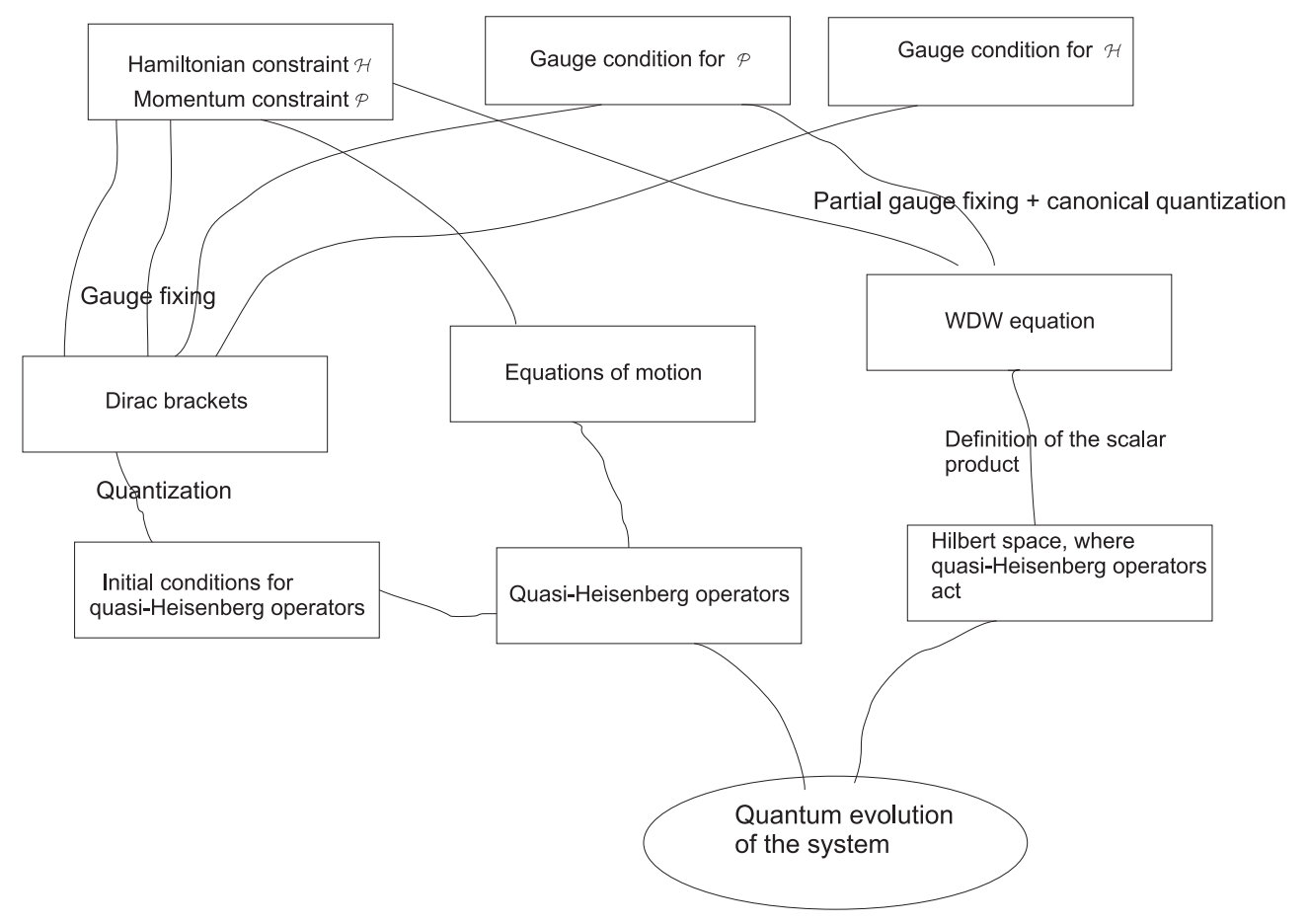

Figure 1. Quasi-Heisenberg quntization scheme

It should be noted that the Heisenberg picture for gravity quantization using anticommutative ghost variables was discussed in Ref. [31]. The Schrödinger picture using anticommutative ghost variables has also been developed [32-34]. It would be instructive to compare these approaches with one another and with the quasi-Heisenberg for some simple minisuperspace model, but this has not been done yet. The Schrödinger equation was also used in a quiet different content [35] to describe distribution of the universe stricture elements such as galaxies, clusters, superclusters [36] or dark matter particles over scale factor (red shift) [37].

The aim of the present work is to consider more closely the setting of the initial conditions for the quasi-Heisenberg operators in connection with the singularity problem. 
Though the initial singularity remains, the situation differs substantially from the classic one. It appears, that one may set the initial conditions at the singularity directly. It will be demonstrated by the example of the Gowdy model described in section 2 of the paper. This model admits the analytical solution within the whole time domain and has been used for singularity investigation [38, 39]. Also, this model allows choosing the out-vacuum state, as the gravitational waves evolve against a classical background $^{1}$.

Because the existence or nonexistence of the singularity turns out to be related to the problem of the regularization of the vacuum energy $[38,39]$, the issue of vacuum energy is briefly discussed in section 3 , where the evolution of the system in a vacuum state is considered and then compared with the evolution in the state given by the wave packet used in section 2 .

\section{Quasi-Heisenberg Quantization of the Gowdy Model}

The polarized $\mathbf{T}^{3}$ Gowdy model corresponds to an anisotropic universe, where the gravitational waves travel unidirectionally. Let us take a metric in the form of

$$
d s^{2}=e^{\tau-\lambda}\left(d \eta^{2}-d x^{2}\right)-e^{2 \tau+2 \sqrt{3} V} d y^{2}-e^{2 \tau-2 \sqrt{3} V} d z^{2},
$$

where the coordinates $\eta, x, y, z$ define points of the Pseudo-Rimanian manifold. Quantities $\tau, \lambda$ and $V$ determine the manifold metric and depend on the variables $\eta$ and $x$ only, which takes the values at $\{0, \infty\}$ and $\{0,2 \pi\}$ respectively. We treat the coordinate $\eta$ as a "time"-parameter describing the evolution of a system.

In Eq. (1) we use a slightly different gauge than the original Gowdy's one:

$$
d s^{2}=e^{-\lambda+3 \tau} d t^{2}-e^{-\lambda-\tau} d X^{2}-e^{2 \tau+2 \sqrt{3} V} d y^{2}-e^{2 \tau-2 \sqrt{3} V} d z^{2}
$$

where $d t=e^{-\tau} d \eta$ and $d X=e^{\tau} d x$. The motivation is that in the gauge given by (1), the equations of motion contain a difference of the potential and kinetic energies of field oscillators. In the absence of evolution, this quantity is zero by virtue of the virial theorem. When the system evolves, the virial theorem is violated [43]. As was shown earlier, the difference of the potential and kinetic energies provides a value of the universe acceleration parameter for the Friedman universe which is comparable with the observed one [44].

The Einstein equations lead to three equations of motion

$$
\begin{gathered}
V^{\prime \prime}-\partial_{x x} V+2 \tau^{\prime} V^{\prime}-\partial_{x} V \partial_{x} \tau=0, \\
\tau^{\prime \prime}-\partial_{x x} \tau=2\left(\partial_{x} \tau\right)^{2}-2\left(\tau^{\prime}\right)^{2}, \\
\lambda^{\prime \prime}-\partial_{x x} \lambda=4\left(\partial_{x} \tau\right)^{2}-4\left(\tau^{\prime}\right)^{2}-6\left(\partial_{x} V\right)^{2}+6\left(V^{\prime}\right)^{2},
\end{gathered}
$$

and two constraints

$$
\begin{aligned}
\mathcal{H}(\eta, x)=e^{2 \tau}\left(\frac{1}{3}\left(\partial_{x} \tau\right)^{2}+\frac{1}{2}\left(\partial_{x} V\right)^{2}+\frac{1}{6} \partial_{x} \tau \partial_{x} \lambda+\frac{1}{3} \partial_{x x} \tau-\frac{1}{3} \tau^{\prime 2}+\frac{1}{2} V^{\prime 2}+\frac{1}{6} \tau^{\prime} \lambda^{\prime}\right) & =0 \\
\mathcal{P}(\eta, x)=e^{2 \tau}\left(\frac{1}{6} \partial_{x} \lambda \tau^{\prime}+\partial_{x} V V^{\prime}+\frac{1}{6} \partial_{x} \tau \lambda^{\prime}+\frac{1}{3} \partial_{x} \tau^{\prime}\right) & =0,
\end{aligned}
$$

where prime denotes differentiation over time $\eta$.

Let us discuss the structure of the equations of motion (2)-(4). Eqs. (2)-(4) contain a part corresponding to the wave equation. The remaining parts belong to two different types. The first one is of $\left(\tau^{\prime} V^{\prime}-\partial_{x} V \partial_{x} \tau\right)-$ type. In this case, we refer to $V$ as a "field" variable, whereas $\tau$ plays a role of the "background" against which the field $V$ oscillates. The equations for the "background" variable contain the difference of the kinetic and potential energies, e.g., $\left(\tau^{\prime}\right)^{2}-\left(\partial_{x} \tau\right)^{2}$ or $\left(V^{\prime}\right)^{2}-\left(\partial_{x} V\right)^{2}$. The situation is analogous to the model representing a string against a curved background [29]. However, the equations for the background

\footnotetext{
1 In the general case quasi-Heisenberg picture admits quantum background.
} 
variable $\tau$ differ from those considered in Ref. [29], because Eq. (3) for $\tau$ is isolated, whereas the field variables contribute to the corresponding equation for the "background" in the toy model [29]. On the other hand, there is another "background" variable $\lambda$ here, because the Gowdy model is anisotropic, and one needs two variables $\tau$ and $\lambda$ to describe the background. It should be noted that the "background" variable $\lambda$ does not influence the oscillations of the "field" $V$.

In a general case, an inhomogeneous variable $\tau$ has to be treated as quantum operator with the related algebra. However, the goal of the present paper is to consider the initial conditions near singularity. Thus, for simplicity, a particular gauge is taken where $\tau$ is non-quantum (i.e., "c"-number valued) and spatially homogeneous. That results in the solution akin to the Gowdy one [40-42].

It is convenient to expand the dynamical variables into the Fourier series

$$
\begin{aligned}
V(\eta, x) & =\sum_{k=1} \mathcal{V}_{k}(\eta) e^{i k x}, \\
\lambda(\eta, x) & =\sum_{k=1} \Lambda_{k}(\eta) e^{i k x}, \\
\tau(\eta, x) & =\sum_{k=1} T_{k}(\eta) e^{i k x} .
\end{aligned}
$$

The equation of motion (3) for $\tau$ is isolated from others. Thus, the spatially uniform initial conditions for $\tau$ make it spatially independent in the course of evolution. So one can take the initial conditions

$$
T_{k}(0)=\delta_{0, k} \mathcal{T}_{0}, \quad T_{k}^{\prime}(0)=\delta_{0, k} e^{-2 \mathcal{T}_{0}} \Pi,
$$

where $\Pi$ and $\mathcal{T}_{0}$ are some constants. We shall further refer to $T_{0}(\eta)$ as $\tau(\eta)$.

Advancing in such a way and using the aforementioned gauge, one comes to the following equations of motion and constraints:

$$
\begin{gathered}
\tau^{\prime \prime}+2\left(\tau^{\prime}\right)^{2}=0, \\
\mathcal{V}_{k}^{\prime \prime}+k^{2} \mathcal{V}_{k}+2 \tau^{\prime} \mathcal{V}_{k}^{\prime}=0, \\
\Lambda_{0}^{\prime \prime}=-4\left(\tau^{\prime}\right)^{2}+6 \sum_{q} \mathcal{V}_{q}^{\prime} \mathcal{V}_{-q}^{\prime}-q^{2} \mathcal{V}_{q} \mathcal{V}_{-q}, \\
\mathcal{H}_{k}=e^{2 \tau}\left(-\delta_{k, 0} \frac{1}{3} \tau^{\prime 2}+\frac{1}{6} \tau^{\prime} \Lambda_{k}^{\prime}+\frac{1}{2} \sum_{q} \mathcal{V}_{q}^{\prime} \mathcal{V}_{k-q}^{\prime}-q(k-q) \mathcal{V}_{q} \mathcal{V}_{k-q}\right)=0, \\
\mathcal{P}_{k}=e^{2 \tau}\left(\frac{1}{6} i k \Lambda_{k} \tau^{\prime}+\sum_{q}(i q) \mathcal{V}_{q} \mathcal{V}_{k-q}^{\prime}\right)=0 .
\end{gathered}
$$

The equations of motion (9)-(11) can be obtained from the Hamiltonian $H=\mathcal{H}_{0}$. It should be noted that $\Lambda_{k}$ at $k \neq 0$ is completely defined by the momentum constraint equation (13), namely

$$
\Lambda_{k}=-\frac{6}{k \tau^{\prime}} \sum_{q} q \mathcal{V}_{q} \mathcal{V}_{k-q}^{\prime}
$$

which reduces the system to $\tau, \Lambda_{0}, \mathcal{V}_{k}$.

One can introduce the momenta

$$
\begin{array}{r}
\pi_{k}=\frac{\partial H}{\partial \mathcal{V}_{k}^{\prime}}=e^{2 \tau} \mathcal{V}_{-k}^{\prime}, \quad P_{\Lambda}=\frac{\partial H}{\partial \Lambda_{0}^{\prime}}=e^{2 \tau} \tau^{\prime} / 6, \\
P_{\tau}=-\frac{\partial H}{\partial \tau^{\prime}}=e^{2 \tau}\left(\frac{2}{3} \tau^{\prime}-\Lambda_{0}^{\prime} / 6\right),
\end{array}
$$

and rewrite the Hamiltonian in terms of these momentums

$$
H=e^{-2 \tau}\left(-6 P_{\Lambda} P_{\tau}+12 P_{\Lambda}^{2}+\frac{1}{2} \pi_{0}^{2}+\sum_{k \geq 1} \pi_{k} \pi_{k}^{*}\right)+e^{2 \tau} \sum_{k \geq 1} k^{2} \mathcal{V}_{k} \mathcal{V}_{k}^{*},
$$


where it is taken into account that $\pi_{-k}=\pi_{k}^{*}$ and $\mathcal{V}_{-k}=\mathcal{V}_{k}^{*}$.

The quasi-Heisenberg quantization consists in quantization of the equations of motion [28-30]. Briefly, this procedure can be described in the following way. The operator initial conditions for the equations of motion include the conditions (8) rewritten in terms of $\tau$ and the remaining conditions

$$
\begin{gathered}
\hat{\mathcal{V}}_{k}(0)=\hat{v}_{k}, \quad \hat{\mathcal{V}}_{k}^{\prime}(0)=e^{-2 \mathcal{T}_{0}} \hat{p}_{-k}, \quad \hat{\Lambda}_{0}(0)=L_{0}, \\
\hat{\Lambda}_{0}^{\prime}(0)=e^{-2 \mathcal{T}_{0}}\left(24 P_{\Lambda}(0)-6 \hat{P}_{\tau}(0)\right), \quad \tau(0)=\mathcal{T}_{0}, \quad \tau^{\prime}(0)=6 e^{-2 \mathcal{T}_{0}} P_{\Lambda}(0),
\end{gathered}
$$

where $\mathcal{T}_{0}$ and $L_{0}$ are some $c$-numbers,

$$
\begin{aligned}
P_{\Lambda}(0) & =\Pi \\
\hat{P}_{\tau}(0) & =\frac{1}{6 \Pi}\left(12 \Pi^{2}+\frac{1}{2} \sum_{k} \hat{p}_{k} \hat{p}_{-k}+e^{4 \mathcal{T}_{0}} k^{2} \hat{v}_{k} \hat{v}_{-k}\right),
\end{aligned}
$$

and $\Pi$ is the $c$-number as well. The operators $\hat{p}_{k}$ and $\hat{v}_{k}$ do not depend on time and satisfy the standard commutation relations $\left[\hat{p}_{k}, \hat{v}_{k^{\prime}}\right]=-i \delta_{k, k^{\prime}}$, where $\delta_{k, k^{\prime}}$ is the Kronneker symbol. They are initial values of the time-dependent operators $\hat{\pi}_{k}(\eta)$ and $\hat{\mathcal{V}}_{k}(\eta)$. One may implement the above operator commutation relations by the representation $\hat{v}_{k}=v_{k}, \hat{p}_{k}=-i \frac{\partial}{\partial v_{k}}$, or by the representation $\hat{p}_{k}=p_{k}, \hat{v}_{k}=i \frac{\partial}{\partial p_{k}}$. Thus, one has the following commutator algebra at the initial moment of time $\left[\hat{\pi}_{k}, \hat{\mathcal{V}}_{k^{\prime}}\right]=-i \delta_{k, k^{\prime}}$,

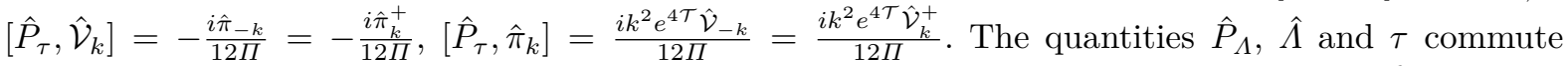
with all others initially. The commutator algebra could be also obtained with the help of the Dirac brackets $[29,30]$.

After the definition of initial conditions for the operator evolution (Eq. (18), see Fig. 1), the following step is to define the Hilbert space where the quasi-Heisenberg operators act. As we stated previously, the quasi-Heisenberg picture is an alternative to the WDW equation, however, it turns out that for building the Hilbert space, one should return to the Hamiltonian (16) and consider it as the WDW equation in the vicinity of $\mathcal{T}_{0} \rightarrow-\infty$ [28-30]. Heretofore, the momentum $P_{\Lambda}$ should be excluded with the help of the gauge condition $P_{\Lambda}=\Pi$.

The corresponding WDW equation in the vicinity of $\tau=\mathcal{T}_{0} \rightarrow-\infty$ is given as

$$
\left(-i 6 \Pi \frac{\partial}{\partial \tau}+12 \Pi^{2}-\frac{1}{2} \frac{\partial^{2}}{\partial v_{0}^{2}}-\sum_{k \geq 1} \frac{\partial}{\partial v_{k}} \frac{\partial}{\partial v_{k}^{*}}\right) \Psi\left(\tau, . . v_{1}^{*}, v_{0}, v_{1} \ldots\right)=0
$$

where term $e^{4 \tau} k^{2} \hat{v}_{k} \hat{v}_{k}^{+}$is omitted because the states of the form of the wave packet will be considered below. Let in some of this states typical value of the square of momentum of the mode $k$ is $<\hat{p}_{k} \hat{p}_{k}^{+}>\sim 1 / a_{k}$, then the typical value of $<e^{4 \tau} k^{2} \hat{v}_{k} \hat{v}_{k}^{+}>\sim e^{4 \tau} k^{2} a_{k}$ due to uncertainty principle, so it becomes negligible in the vicinity $\tau=\mathcal{T}_{0} \rightarrow-\infty$ which just be needed. Here $\hat{v}_{k}^{+}=v_{k}^{*}, \hat{p_{k}^{+}}=-i \frac{\partial}{\partial v_{k}^{*}}$.

The mean value of the quasi-Heisenberg operator $A\left(\eta, \tau, v_{i}, \hat{p}_{i}\right)$ is given by formula

$$
<\psi\left|\hat{A}\left(\eta, \tau, v_{j},-i \frac{\partial}{\partial v_{j}}\right)\right| \psi>=\left.\int \Psi^{*}\left(\tau, v_{j}\right) \hat{A}\left(\eta, \tau, v_{j},-i \frac{\partial}{\partial v_{j}}\right) \Psi\left(\tau, v_{j}\right) d v_{0} d v_{1} d v_{1}^{*} \ldots\right|_{\tau=\mathcal{T}_{0} \rightarrow-\infty},
$$

where the integral over $d z d z^{*} \equiv \frac{\rho d \rho d \phi}{2 \pi i}$ and $z=\rho e^{i \phi}$ is understood in the holomorphic representation [45]. It should be noted that as well as in the Klein-Gordon current scalar product [28-30] there is no integration over the variable $\tau$ in equation (20). Instead, it is set to some quantity $\mathcal{T}_{0}$. For instance, in more a general case of the equation containing the derivatives $\frac{\partial^{2}}{\partial \tau^{2}}$ as well as $\frac{\partial}{\partial \tau}$, the scalar product should contain as the term $i\left(\frac{\partial \Psi}{\partial \tau} \Psi^{*}-\frac{\partial \Psi^{*}}{\partial \tau} \Psi\right)$ of the "current" type, so the term $\Psi^{*} \Psi$ of the "density" type. In any case the quantity $\tau$ should be set to some value $\mathcal{T}_{0}$ [46]. Here, the quantity $\mathcal{T}_{0}$ is chosen to be initially finite, thus avoiding the singularity, but finally the limit $\mathcal{T}_{0} \rightarrow-\infty$ is taken.

The general solution of Eq. (19) may be written in the form of the wave packet 


$$
\begin{array}{r}
\Psi\left(\tau, . . v_{1}^{*}, v_{0}, v_{1} \ldots\right)=\int C\left(. . p_{1}^{*}, p_{0}, p_{1} \ldots\right) \exp \left(-\frac{i}{6 \Pi}\left(12 \Pi^{2}+\frac{1}{2} p_{0}^{2}\right.\right. \\
\left.\left.+\sum_{k \geq 1} p_{k} p_{k}^{*}\right) \tau+i \sum_{k \geq 0} v_{k} p_{k}^{*}\right) d p_{0} d p_{1} d p_{1}^{*} \ldots
\end{array}
$$

In the momentum representation, the wave function (21) takes the form

$$
\psi\left(\tau, . . p_{1}^{*}, p_{0}, p_{1} \ldots\right)=C\left(. . p_{1}^{*}, p_{0}, p_{1} \ldots\right) \exp \left(-\frac{i}{6 \Pi}\left(12 \Pi^{2}+\frac{1}{2} p_{0}^{2}+\sum_{k \geq 1} p_{k} p_{k}^{*}\right) \tau\right)
$$

and formula (20) for mean value looks like

$$
<\psi\left|\hat{A}\left(\eta, \tau, \mathrm{i} \frac{\partial}{\partial p_{j}}, p_{j}\right)\right| \psi>=\left.\int \psi^{*}\left(\tau, p_{j}\right) \hat{A}\left(\eta, \tau, \mathrm{i} \frac{\partial}{\partial p_{j}}, p_{j}\right) \psi\left(\tau, p_{j}\right) d p_{0} d p_{1} d p_{1}^{*} \ldots\right|_{\tau=\mathcal{T}_{0} \rightarrow-\infty} .
$$

For this simple model, the analytical solution exists that allows demonstrating the calculation of mean values in detail. The solution of Eq. (9) is

$$
\tau(\eta)=\mathcal{T}_{0}+\frac{1}{2} \ln \left(1+12 \Pi e^{-2 \mathcal{T}_{0}} \eta\right)
$$

First, let us consider the solution of Eq. (10) in the vicinity of $\tau \sim \mathcal{T}_{0} \rightarrow-\infty$. It takes the form

$$
\hat{\mathcal{V}}_{k}(\eta) \approx \hat{v}_{k}+\frac{1}{12 \Pi} p_{k}^{*} \ln \left(1+12 \Pi e^{-2 \mathcal{T}_{0}} \eta\right)
$$

If $\mathcal{T}_{0}$ tends to minus infinity, then the expression (24) for $\tau(\eta)$ becomes $\tau(\eta)=\frac{1}{2} \ln (12 \Pi \eta)$. However, the expression for the operator $\hat{\mathcal{V}}_{k}(\eta)$ diverges formally as $\mathcal{T}_{0} \rightarrow-\infty$. This reflects the fact that it is impossible to set the field values at the singularity in the classical picture. Below we demonstrate that the quantum picture validates the limit of $\mathcal{T}_{0} \rightarrow-\infty$ for the mean observable values.

Let us consider the mean value of (25) over the wave packet (22)

$$
\begin{array}{r}
<\psi\left|\hat{\mathcal{V}}_{k}\right| \psi>=\int\left(C\left(. . p_{1}^{*}, p_{0}, p_{1} \ldots\right)\right)^{*} \exp \left(\frac{i}{6 \Pi}\left(12 \Pi^{2}+\sum_{q \geq 0} p_{q} p_{q}^{*}\right) \mathcal{T}_{0}\right) \\
\left(i \frac{\partial}{\partial p_{k}}+\frac{1}{12 \Pi} p_{k}^{*} \ln \left(1+12 \Pi e^{-2 \mathcal{T}_{0}} \eta\right)\right) \exp \left(-\frac{i}{6 \Pi}\left(12 \Pi{ }^{2}+\sum_{q \geq 0} p_{q} p_{q}^{*}\right) \mathcal{T}_{0}\right) \\
\left.C\left(. . p_{1}^{*}, p_{0}, p_{1} \ldots\right) d p_{0} d p_{1} d p_{1}^{*} \ldots\right|_{\mathcal{T}_{0} \rightarrow-\infty} \\
=\int\left(C\left(. . p_{1}^{*}, p_{0}, p_{1} \ldots\right)\right)^{*}\left(\frac{1}{12 \Pi} p_{k}^{*} \ln \left(1+12 \Pi e^{-2 \mathcal{T}_{0}} \eta\right)+\frac{1}{6 \Pi} p_{k}^{*} \mathcal{T}_{0}+i \frac{\partial}{\partial p_{k}}\right) \\
\left.=\int\left(C\left(. . p_{1}^{*}, p_{0}, p_{1} \ldots\right)\right)^{*}\left(\frac{1}{12 \Pi} p_{k}^{*} \ln (12 \Pi \eta)+i \frac{\partial}{\partial p_{k}}\right) C\left(. . p_{1}^{*}, p_{0}, p_{1} \ldots\right) d p_{0} d p_{1} d p_{1}^{*} \ldots\right)\left.d p_{0} d p_{1} d p_{1}^{*} \ldots\right|_{\mathcal{T}_{0} \rightarrow-\infty}
\end{array}
$$

One can see from Eq. (26) that the divergent terms with $\mathcal{T}_{0} \rightarrow-\infty$ cancel each other, and the mean value of $\hat{\mathcal{V}}_{k}$ is finite. Hence, the wave packet defined at the singularity determines the entire evolution of the system. 
The approximate expression for $\hat{\mathcal{V}}_{k}$ has been used above. It is valid for $\eta \sim 0$. However, it is intensional to consider the exact expression and the contribution of $V$ - quantum fluctuations to the $\lambda-$ evolution. The exact solution of the equation of motion (10) with $\tau(\eta)$ given by (24) takes the form

$$
\begin{aligned}
\hat{\mathcal{V}}_{k}(\eta)= & \frac{\pi}{24 \Pi}\left(p_{k}^{*} J_{0}\left(\frac{e^{2 \mathcal{T}_{0}} k}{12 \Pi}\right) Y_{0}\left(k\left(\eta+\frac{e^{2 \mathcal{T}_{0}}}{12 \Pi}\right)\right)-J_{0}\left(k\left(\eta+\frac{e^{2 \mathcal{T}_{0}}}{12 \Pi}\right)\right)\right. \\
& \left.\left(p_{k}^{*} Y_{0}\left(\frac{e^{2 \mathcal{T}_{0}} k}{12 \Pi}\right)+k e^{2 \mathcal{T}_{0}} \hat{v}_{k} Y_{1}\left(\frac{e^{2 \mathcal{T}_{0}} k}{12 \Pi}\right)\right)+k e^{2 \mathcal{T}_{0}} \hat{v}_{k} J_{1}\left(\frac{e^{2 \mathcal{T}_{0}} k}{12 \Pi}\right) Y_{0}\left(k\left(\eta+\frac{e^{2 \mathcal{T}_{0}}}{12 \Pi}\right)\right)\right) .
\end{aligned}
$$

Here $J_{0}(z), Y_{0}(z), Y_{1}(z)$ and $J_{1}(z)$ are the Bessel functions. The second derivative of $\Lambda_{0}$ can be determined from the equation of motion (11), whereas its first derivative can be determined from the Hamiltonian constraint (12):

$$
\hat{\Lambda}_{0}^{\prime}=\frac{1}{\tau^{\prime}}\left(2 \tau^{\prime 2}-3 \hat{\mathcal{V}}_{0}^{\prime 2}-6 \sum_{k \geq 1} \hat{\mathcal{V}}_{k}^{\prime} \hat{\mathcal{V}}_{k}^{\prime+}+k^{2} \hat{\mathcal{V}}_{k} \hat{\mathcal{V}}_{k}^{+}\right)
$$

Here $\hat{\mathcal{V}}_{k}^{+}$should be obtained from Eq. (27) by changing $\hat{v}_{k} \rightarrow{\hat{v_{k}^{+}}}^{+}=i \frac{\partial}{\partial p_{k}^{*}}, p_{k}^{*} \rightarrow p_{k}$. Thus the most intriguing problem is the calculation mean values of $\hat{\mathcal{V}}_{k}^{\prime} \hat{\mathcal{V}}_{k}^{\prime+}$ and $k^{2} \hat{\mathcal{V}}_{k} \hat{\mathcal{V}}_{k}^{+}$, which are constituents of Eqs. (11) and (28) for $\hat{\Lambda}_{0}^{\prime}, \hat{\Lambda}_{0}^{\prime \prime}$. Tracing these quantities allows calculating the $\hat{\Lambda}_{0}$-evolution.

Let us take the Gaussian form of the wave packet to determine the evolution of the system

$$
C\left(. . p_{1}^{*}, p_{0}, p_{1} \ldots\right)=\prod_{k=0}^{\infty} N_{k} \exp \left(-a_{k} p_{k} p_{k}^{*}\right)
$$

where the constant $a_{k}$ determines the width of the packet for each mode and $N_{k}$ is the normalization factor. The calculation according to (23) leads to the expressions defining the mean value of the potential energy $\Xi_{k}$ and the value of the kinetic energy $K_{k}$ of each mode $k \neq 0$ :

$$
\begin{aligned}
\Xi_{k} \equiv & <\psi\left|k^{2} \hat{\mathcal{V}}_{k} \hat{\mathcal{V}}_{k}^{+}\right| \psi>=\frac{k^{2}}{1152 a_{k} \Pi^{2}}\left(\left(4 J _ { 0 } ^ { 2 } ( k \eta ) \left(144 a_{k}^{2} \Pi^{2}+\log ^{2}\left(\frac{k}{24 \Pi}\right)\right.\right.\right. \\
& \left.\left.\left.+2 \gamma \log \left(\frac{k}{24 \Pi}\right)+\gamma^{2}\right)-4 \pi\left(\log \left(\frac{k}{24 \Pi}\right)+\gamma\right) J_{0}(k \eta) Y_{0}(k \eta)+\pi^{2} Y_{0}^{2}(k \eta)\right)\right) \\
K_{k} & \equiv<\psi\left|\hat{\mathcal{V}}_{k}^{\prime} \hat{\mathcal{V}}_{k}^{\prime+}\right| \psi>=\frac{k^{2}}{1152 a_{k} \Pi^{2}}\left(\left(4 J _ { 1 } ^ { 2 } ( k \eta ) \left(144 a_{k}^{2} \Pi^{2}+\log ^{2}\left(\frac{k}{24 \Pi}\right)\right.\right.\right. \\
& \left.\left.\left.+2 \gamma \log \left(\frac{k}{24 \Pi}\right)+\gamma^{2}\right)-4 \pi\left(\log \left(\frac{k}{24 \Pi}\right)+\gamma\right) J_{1}(k \eta) Y_{1}(k \eta)+\pi^{2} Y_{1}^{2}(k \eta)\right)\right)
\end{aligned}
$$

where $J_{0}(z), Y_{0}(z), J_{1}(z)$ and $Y_{1}(z)$ are the Bessel functions and $\gamma$ is the Euler constant.

A spatially uniform mode contains only the kinetic energy term

$$
\left.K_{0} \equiv \frac{1}{2}<\psi\left|\mathcal{V}_{0}^{\prime 2}\right| \psi\right\rangle=\frac{1}{1152 a_{0}^{2} \Pi^{2} \eta^{2}}
$$

For further analysis, it is convenient to consider the quasi-classical sector corresponding to late times. This insight can be provided by expanding the Bessel function into series over a large argument and 
keeping the leading terms:

$$
\begin{gathered}
Y_{0}(z) \approx \frac{1}{\sqrt{\pi z}}\left(\left(-\frac{9}{128 z^{2}}-\frac{1}{8 z}+1\right) \sin (z)+\left(\frac{9}{128 z^{2}}-\frac{1}{8 z}-1\right) \cos (z)\right) \\
J_{0}(z) \approx \frac{1}{\sqrt{\pi z}}\left(\left(-\frac{9}{128 z^{2}}+\frac{1}{8 z}+1\right) \sin (z)+\left(-\frac{9}{128 z^{2}}-\frac{1}{8 z}+1\right) \cos (z)\right) \\
J_{1}(z) \approx \frac{1}{\sqrt{\pi z}}\left(\left(\frac{15}{128 z^{2}}+\frac{3}{8 z}+1\right) \sin (z)+\left(-\frac{15}{128 z^{2}}+\frac{3}{8 z}-1\right) \cos (z)\right) \\
Y_{1}(z) \approx \frac{1}{\sqrt{\pi z}}\left(\left(-\frac{15}{128 z^{2}}+\frac{3}{8 z}-1\right) \sin (z)+\left(-\frac{15}{128 z^{2}}-\frac{3}{8 z}-1\right) \cos (z)\right) .
\end{gathered}
$$

Then, a simple estimation results from replacement the oscillating multipliers by their time-averaged values as $\cos ^{2}(k \eta) \rightarrow \frac{k}{2 \pi} \int_{0}^{2 \pi / k} \cos ^{2}(k \eta) d \eta=\frac{1}{2}, \sin ^{2}(k \eta) \rightarrow \frac{1}{2}$, and $\sin (k \eta) \cos (k \eta) \rightarrow 0$.

Using Eqs. (11) and (28) we get

$$
\begin{aligned}
<\psi\left|\hat{\Lambda}_{0}^{\prime}\right| \psi> & \approx \frac{1}{\eta}\left(1-\frac{1}{96 a_{0} \Pi^{2}}\right)-\sum_{k \geq 1} \frac{k F_{k}}{6 \Pi^{2} \pi a_{k}}+\frac{12 a_{k} k}{\pi}+\frac{1}{\eta^{2}}\left(\frac{F_{k}}{48 \pi \Pi^{2} k a_{k}}+\frac{3 a_{k}}{2 \pi k}\right), \\
& <\psi\left|\hat{\Lambda}_{0}^{\prime \prime}\right| \psi>\approx-\frac{1}{\eta^{2}}\left(1-\frac{1}{96 a_{0} \Pi^{2}}\right)+\frac{1}{\eta^{3}} \sum_{k \geq 1} \frac{F_{k}}{24 \pi \Pi^{2} k a_{k}}+\frac{3 a_{k}}{\pi k},
\end{aligned}
$$

where $F_{k}=\left(\frac{\pi^{2}}{8}+\frac{\gamma^{2}}{2}+\frac{1}{2} \ln ^{2}\left(\frac{k}{24 \Pi}\right)+\gamma \ln \left(\frac{k}{24 \Pi}\right)\right)$.

It should be noted that Eq. (32) describing the averaged second derivative of $\Lambda_{0}$ in a sense of the time-averaged evolution can be obtained from Eq. (31) by the differentiation over $\eta$. Turning to a continuous limit of $\sum_{k} \rightarrow \frac{1}{2 \pi} \int d k$, we can see that the second term in Eq. (31), corresponding to the vacuum energy, diverges for any asymptotic of $a_{k}$ at large $k$.

The most divergent term $\frac{k F_{k}}{6 \Pi^{2} \pi a_{k}}+\frac{12 a_{k} k}{\pi}$ vanishes under differentiation of Eq. (31). The remained term is the mean value of the difference of the potential and kinetic energies of field oscillators, and has been considered in Ref. [44] for the Friedman universe. It has been found that this term defines the value of the acceleration parameter of universe, which is compatible with the observed one. One has noted, that the UV cut-off of momenta was used for the estimates [44] for the Friedman universe. The present-day universe expands isotropically, so one cannot compare the results of the above calculations with some observational values directly. The early stages of the universe could be highly anisotropic [47]. Particle creation during the anisotropic cosmological expansion and its back reaction to the metric have been considered [48]. It is interesting that the authors of Ref. [48] faced the necessity to set initial conditions for the evolution. They were forced to begin the evolution from a certain artificial moment of time. As we have seen above in the quasi-Heisenberg picture there exists fundamental possibility to set the initial conditions at the singularity itself and therefore to improve the analysis of Ref. [48].

\section{Evolution Determined by the Vacuum State}

In the considered gauge the background variable $\tau$ is not quantum. For this particular case, one can use the ordinary quantization using the creation and annihilation operators. Thus, we consider the quantization of the field $V$ against the time-dependent background $\tau(\eta)=\frac{1}{2} \ln (12 \Pi \eta)$. In this case, the field $V$ is represented as $[1]$

$$
\mathcal{V}_{k}(\eta)=\sum_{k} \hat{\mathrm{a}}_{k} u_{k}(\eta)+\hat{\mathrm{a}}_{k}^{+} u_{k}^{*}(\eta),
$$

where $\left[\hat{\mathrm{a}}_{k}, \hat{\mathrm{a}}_{k}^{+}\right]=1$. 
The function $u_{k}(\eta)$ should satisfy the condition

$$
e^{2 \tau(\eta)}\left(u_{k}^{*}(\eta) u_{k}^{\prime}(\eta)-u_{k}(\eta) u_{k}^{* \prime}(\eta)\right)=i
$$

The mean values of the kinetic and potential energies of the mode $k$ in a vacuum state equal to

$$
\Xi_{k}=<0\left|k^{2} \mathcal{V}_{k} \mathcal{V}_{k}^{+}\right| 0>=k^{2} u_{k}^{*} u_{k}, \quad K_{k}=<0\left|\mathcal{V}_{k}^{\prime} \mathcal{V}_{k}^{+\prime}\right| 0>=u_{k}^{* \prime} u_{k}^{\prime}
$$

Thus, one has to determine the functions $u_{k}$. The vacuum state is defined as a state vanishing under the action of the annihilation operator: $\hat{\mathrm{a}}_{k} \mid 0>=0$. However, the definition of $u_{k}$ is ambiguous. It should be noted that there exists a family of functions $u_{k}$ which satisfy Eq. (34) and are interrelated by the Bogolubov's transformation. It was shown [49] the vacuum state could be defined through the minimization of some functional containing the difference of the potential and kinematic energies of field oscillators. In such a way one comes to the function

$$
u_{k}(\eta)=\frac{1}{4} \sqrt{\frac{\pi}{3 \Pi}} H_{0}^{(2)}(|k| \eta)
$$

where $H_{0}^{(2)}(z)$ is the Hankel function of the second kind. There is no particle (i.e., graviton) creation here, because the difference of the kinetic and potential energies is not an oscillating quantity [49].

Using the asymptotics of the Hankel function for large arguments,

$$
H_{0}^{(2)}(z) \approx \sqrt{\frac{2}{\pi z}} e^{-i(z-\pi / 4)}\left(1+\frac{i}{8 z}-\frac{9}{128 z^{2}}\right)
$$

one can obtain for the mean values of $\hat{\Lambda}_{0}^{\prime}$ and $\hat{\Lambda}_{0}^{\prime \prime}$ over vacuum state

$$
\begin{aligned}
<0\left|\hat{\Lambda}_{0}^{\prime}\right| 0>\approx \frac{1}{\eta}-\sum_{k \geq 1} \frac{k}{\Pi}+\frac{1}{8 k \eta^{2} \Pi}, \\
<0\left|\hat{\Lambda}_{0}^{\prime \prime}\right| 0>\approx-\frac{1}{\eta^{2}}+\sum_{k \geq 1} \frac{1}{4 k \eta^{3} \Pi} .
\end{aligned}
$$

It is interesting to compare the above results with those from the quasi-Heisenberg quantization. For this aim one has to find the value $a_{k}$ in Eqs. (31),(32) which minimizes the constant part contribution $\frac{k F_{k}}{6 \Pi^{2} \pi a_{k}}+\frac{12 a_{k} k}{\pi}$ of every mode to $\Lambda_{0}^{\prime}$ given by Eq. (31). That gives $a_{k}=\frac{1}{6 \Pi} \sqrt{\frac{F_{k}}{2}}$. Substitution of this value into Eqs. (31) and (32) leads to

$$
\begin{gathered}
<\psi\left|\hat{\Lambda}_{0}^{\prime}\right| \psi>\approx \frac{1}{\eta}\left(1-\frac{1}{96 a_{0} \Pi^{2}}\right)-\sum_{k \geq 1} \sqrt{1+\frac{4}{\pi^{2}}\left(\gamma+\ln \left(\frac{k}{24 \Pi}\right)\right)^{2}}\left(\frac{k}{\Pi}+\frac{1}{8 k \eta^{2} \Pi}\right) \\
<\psi\left|\hat{\Lambda}_{0}^{\prime \prime}\right| \psi>\approx-\frac{1}{\eta^{2}}\left(1-\frac{1}{96 a_{0} \Pi^{2}}\right)+\sum_{k \geq 1} \sqrt{1+\frac{4}{\pi^{2}}\left(\gamma+\ln \left(\frac{k}{24 \Pi}\right)\right)^{2}} \frac{1}{4 k \eta^{3} \Pi} .
\end{gathered}
$$

The comparison with Eq. (37) demonstrates that the non-vanishing term supplements a vacuum state term in the quasi-Heisenberg quantization scheme.

Thus, any momentum wave packet defined at singularity gives an inevitable counterpart corresponding to a matter (in this model "matter" consists of gravitational wave quants). There is no need in "matter creation from nothing" in the quasi-Heisenberg picture, because it exists primordially.

Let us briefly discuss the vacuum energy and its relation to singularity. Before regularization, the expressions for the mean values of $\Lambda_{0}^{\prime}$ and $\Lambda_{0}^{\prime \prime}$ are singular. Regularization of the influence of quantized gravitational waves to a background has been considered [38,39]. The author of Ref. [38] has found that the singularity disappears that occurs because the substraction, that she uses in a regularization, 
affects the classical terms. However, the author of Ref. [39] stated that the singularity still remains. His argumentation is that for coherent states the mean values in classical and quantum pictures must coincide. For this purpose he took an appropriate ordering of the creation and annihilation operators in calculating the mean values. However, it should be noted that the vacuum state is a particular case of the coherent state. Thus, it is not surprising that the vacuum fluctuations do not contribute to evolution (i.e. do not affect the singularity) according to [39].

In the previous section it has been conjectured that a difference of the potential and kinetic energies has a physical meaning if one uses the UV cut-off. It comes from the fact that difference of the potential and kinetic energies of field oscillators gives a value of the universe acceleration compatible with observations [44]. Thus, it seems that only the main divergence (also existing in the Minkowsky space-time) should be subtracted.

\section{Outlook}

As was discussed in the previous section, we cannot say infallibly whether singularity exists or not without a fundamental theory of regularization of the vacuum energy. However, earlier no vacuum energy problem has been found in the toy two dimensional model considering string on the curved background [29], because the cosmological expansion is simply a motion of the string center of mass. Fluctuations, including vacuum ones, do not affect the motion of the string center of mass, i.e. the cosmological expansion. Mathematically, this looks as a compensation of scale factor fluctuations by fluctuations of the matter fields [29].

On the other hand, in GR there exists the Isaacson theorem [50] which states that evolution in the mean is determined by the energy-momentum tensor of excitations (perturbation). Thus, in the theories for which the Isaacson theorem is valid the vacuum energy problem emerges. Roughly, since the Isaacson theorem does not differ the vacuum fluctuations from the excitations under vacuum, the vacuum fluctuations contribute to the mean evolution.

Being capable of solving the vacuum energy problem the theories where the Issacson theorem does not exist, are beyond the GR frameworks. One may assume, that a quantum version of the Isaacson theorem should be developed for GR to differ vacuum and non-vacuum fluctuations. Also, it seems important to investigate the connection of the Isaacson theorem with the conformal invariance of the gravity theories ${ }^{2}$.

To summarize, as it was shown in section 2 , it is possible to describe the universe evolution before regularization by a wave packet definition at singularity regardless a regularization procedure. It should be emphasized that the wave packet determined at the singularity is not only an "informational seed" but it is also responsible for the part of the matter in the universe because the gravitons (and, in the general case, the quants of matter fields) appears inevitably at the late time evolution.

It would be interesting to consider a quantum picture of the general $3+1$ BKL-solution [47] in the framework of the quasi-Heisenberg picture including building of the corresponding wave packet at singularity. This work is in progress [53].

\section{References}

1. N. D. Birrell and P. C. W. Davis, Quantum fields in curved space. Univ. Press, 1982.

2. A. D. Linde, Particle physics and inflationary cosmology. Harwood Academic Publishers, 1990.

3. V. Mukhanov, Physical foundations of cosmology. Univ. Press, 2005.

4. S. Dodelson, Modern cosmology. Academic Press, 2003.

5. A. A. Starobinsky, "A new type of isotropic cosmological model without singularity," Phys. Lett. vol. 91B, no. 1 , pp. 99-102, 1980.

6. A. Guth, "The inflationary universe: a possible solution to the horizon and flatness problem," Phys. Rev. D vol. 23 , no. 1, pp. 347-356, 1981.

7. A. R. Liddle and D. H. Lyth, Cosmological inflation and large-scale structure. Univ. Press, 2000.

8. R. Penrose, "Gravitational collapse and space-time singularities," Phys. Rev. vol. 14, no. 3, pp. 57-59, 1965.

9. R.Geroch, "What is a singularity in general relativity?," Ann. Phys., NY vol. 48, no. 3, pp. 526-540, 1968.

\footnotetext{
${ }^{2}$ Recent interesting example of the conformally invariant theory of gravity have been developed [51,52].
} 
10. S. W. Hawking and R. Penrose, "The singularities of gravitational collapse and cosmology," Phys. Rev. D vol. A314, no. 1519, pp. 529-548, 1970.

11. A. Borde and A. Vilenkin, "Violation of the weak energy condition in inflating spacetimes," Phys. Rev. D vol. 56, no. 2, pp. 717-723, 1997.

12. A. Borde, A. H. Guth and A. Vilenkin, "Inflationary spacetimes are incomplete in past directions," Phys. Rev. Lett. vol. 90, no 15, pp. 151301-4, 2003.

13. J. B. Hartle and S. W. Hawking, "Wave function of the universe," Phys. Rev. D vol. 28, no. 12, pp. 2960-2975, 1983.

14. A. Vilenkin, "Quantum Cosmology and the Initial State of the Universe," Phys. Rev. D vol. 37, no. 4, pp. 888-897, 1988.

15. M. Bojowald, "Dynamical Initial Conditions in Quantum Cosmology," Phys. Rev. Lett.vol. 87, no 12, 121301-4, 2001.

16. M. Bojowald, "Initial Conditions for a Universe," Gen. Rel. Grav. vol. 35, no. 11, pp. 1877-1883, 2003.

17. C. Kiefer, "Quantum geometrodynamics: whence, whither?," Gen. Rel. Grav. vol. 41, no. 4, pp.877-901, 2009.

18. A. V. Minkevich, "Gauge approach to Gravity and regular Big Bang theory," Grav. Cosmol. 12, no. 1, pp. $11-20,2006$.

19. G. Santos, G. Gubitosi and G. Amelino-Camelia, "On the initial singularity problem in rainbow cosmology," J. Cosmol. Astropart. Phys. no. 08, pp. 005, 2015.

20. A. Ashtekar, A. Corichi and P. Singh, "Robustness of key features of loop quantum cosmology," Phys. Rev. $D$ vol. 77 , no. $2,024046-17,2008$.

21. M. Bojowald, Quantum cosmology. A fundamental description of the Universe. Springer, 2011.

22. M. Bojowald and G. M. Paily, "A no-singularity scenario in loop quantum gravity," Class. Quant. Grav. vol. 29, no. 24, pp. 242002-8, 2012.

23. V. Husain and O. Winkler, "On Singularity Resolution in Quantum Gravity," Phys.Rev. D, vol. 69, no. 8, pp. 084016-7, 2004

24. P. Tarrio, M. Fernandez-Mendez and G.A. Mena Marugan, "Singularity avoidance in the hybrid quantization of the Gowdy model," Phys. Rev. D vol. 88, no. 8, pp. 084050-11, 2013.

25. A. Ashtekar and B. Gupt, "Generalized effective description of loop quantum cosmology," Phys. Rev. D, vol. 92, no. 8, pp. 084060-15, 2015.

26. B. S. DeWitt, "Quantum Theory of Gravity. I. The Canonical Theory," Phys. Rev. vol. 160, no. 5, pp.1113-1148, 1967.

27. J. A. Wheeler, "Superspace and nature of quantum geometrodynamics," in Battelle Rencontres. Benjamin, 1968.

28. S. L. Cherkas and V. L. Kalashnikov, "Quantum evolution of the Universe in the constrained quasi-Heisenberg picture: from quanta to classics?," Grav. Cosmol. vol. 12, no. 2-3, pp. 126-129, 2006.

29. S. L. Cherkas and V. L. Kalashnikov, "An inhomogeneous toy-model of the quantum gravity with explicitly evolvable observables," Gen. Rel. Grav. vol. 44, no. 12, pp. 3081-3102, 2012.

30. S. L.Cherkas and V. L. Kalashnikov, "Quantization of the inhomogeneous Bianchi I model: quasi-Heisenberg picture," Nonlin. Phen. Compl. Syst. vol. 18, no. 1, pp. 1-14, 2015. Also available as arXiv: 1302.2229

31. G. Vereshkov and L. Marochnik, "Quantum gravity in Heisenberg representation and self-consistent theory of gravitons in macroscopic spacetime," J. Mod. Phys. vol. 4, no. 2, pp. 285-297, 2013.

32. T. P. Shestakova, "Grounds for Quantum Geometrodynamics in an extended phase space and its cosmological consequences," Grav.Cosmol. vol. 5, no. 4, pp. 297-300, 1999.

33. V. A. Savchenko, T.P. Shestakova and G.M. Vereshkov, "Quantum geometrodynamics in extended phase space - I. Physical problems of interpretation and mathematical problems of gauge invariance," Grav.Cosmol. vol. 7, no. 1, pp. 18-28, 2001.

34. V. A. Savchenko, T. P. Shestakova and G. M.Vereshkov, "Quantum geometrodynamics in extended phase space - II. The Bianchi IX model," Grav.Cosmol. vol. 7, no. 2, pp. 102-116, 2001.

35. N. Rosen, "Quantum mechanics of a miniuniverse" Int.J.Theor.Phys. vol. 32, no. 8, pp. 1435-1440, 1993.

36. S. Capozziello, A. Feoli and G. Lambiase, "Oscillating universes as eigensolutions of cosmological Schrödinger equation" Int. J. Mod. Phys. vol. D 9, no. 2, pp. 143-154, 2000.

37. A. Feoli, "Some predictions of the cosmological Schrödinger equation" Int. J. Mod. Phys. vol. D 12, no. 8, pp. 1475-1486, 2003.

38. B. K. Berger, "Singularity avoidance in the semiclassical Gowdy T3 cosmological model," Phys. Lett. vol. 108B, no. 6, pp. 394-398, 1982.

39. V. Husain, "Quantum effects on the singularity of the Gowdy cosmology," Class. Quant. Grav. vol. 4, no. 6, 1587-1598, 1987.

40. R. H. Gowdy, "Vacuum Spacetimes with Two-parameter Spacelike Isometry Groups and Compact Invariant Hypersurfaces: Topologies and Boundary Conditions," Ann. Phys., NY vol. 83, no. 1, pp. 203-241, 1974. 
41. C. W. Mizner, "A minisuperspace Example: The Gowdy T3 Cosmology," Phys. Rev. vol. 8, no. 10, pp. 3271-3285, 1973

42. B. K. Berger, "Quantum graviton creation in a model universe," Ann. Phys. (NY) vol. 83, no. 2, pp. 458-490, 1974.

43. S. V.Anischenko, "Violation of the virial theorem for the ground state of the time-dependent oscillator," Vestnik Belarus State U., ser. Fiz.-Mat. no. 2, pp. 43-46, 2008. in Russian, posted at http://www.elib.bsu.by/handle/123456789/6085

44. S. L. Cherkas and V. L. Kalashnikov, "Determination of the UV cut-off from the observed value of the Universe acceleration," J. Cosmol. Astropart. Phys. no. 01, pp. 028, 2007.

45. L. D. Faddeev and A. A. Slavnov, Gauge fields: an introduction to quantum theory. Addison-Wesley, 1987. 46. A. Mostafazadeh, "Quantum Mechanics of Klein-Gordon-Type Fields and Quantum Cosmology," Ann. Phys. (NY) vol. 309, no. 1, pp. 1-48, 2004.

47. V. A. Belinskii, I. M. Khalatnikov and E. M. Lifshitz, "Oscillatory approach to a singular point in the relativistic cosmology," Advances in Physics vol. 19, pp. 525-573, 1970.

48. V. N. Lukash and A. A. Starobinsky, "Isotropization of cosmological expansion due to particle production," Sov. Phys.-JETP vol. 39, no. 5, pp. 742-747, 1974.

49. S. V. Anischenko, S.L.Cherkas and V.L. Kalashnikov, "Functional minimization method addressed to the vacuum finding for an arbitrary driven quantum oscillator," Nonlin. Phen. Compl. Syst. vol. 12, no. 1, pp. $16-26,2009$

50. R. A. Isaacson, "Gravitational Radiation in the Limit of High Frequency. I. The Linear Approximation and Geometrical Optics," Phys. Rev. vol. 166, no. 5, pp. 1263-1271, 1968.

51. H. Gomes, S. Gryb and T. Koslowski, "Einstein gravity as a 3D conformally invariant theory," Class.Quant.Grav. vol. 28, no. 4, pp. 045005-24, 2011.

52. L. Smolin, "Linking shape dynamics and loop quantum gravity," Phys. Rev. D vol. 90, no. 4, pp. 044070-10 (2014)

53. S. L. Cherkas and V.L. Kalashnikov, "Solution of the discrete Wheeler-DeWitt equation in the vicinity of small scale factors and quantum mechanics in the space of constant negative curvature," Dokl. Akad. Nauk. Belarus vol. 58, no. 2, pp. 45-50 (2014). Also availubal at arXiv: 1406.5837. 